\title{
Huge Extrapleural Hematoma Initially Diagnosed as Massive Hemothorax
}

\author{
Yueh-Feng Tsai ${ }^{\mathrm{a}, \mathrm{c}}$, Chih-Cheng Lu ${ }^{\mathrm{b}}$
}

\begin{abstract}
We report a 50-year-old man who presented with left chest pain after blunt thoracic trauma. A chest radiograph showed a fracture of the left 4th rib. Three days later, worsening chest pain and shortness of breath occurred. A repeated chest X-ray showed a complete opacity of the left hemithorax. The patient was reported to have a massive left hemothorax leading to a shift of the mediastinum to the right. Tube thoracostomy was performed immediately. However, only a small amount of blood-like fluid was drained and the left hemithorax still appeared as a near total opacity on the followup chest radiograph. These findings were interpreted as a massive clotted hemothorax. Surprisingly, an emergency video-assisted thoracic surgery (VATS) revealed no traumatic pleural collections but a huge pleural mass containing a large amount of dark, reddish blood clots. The blood clots were evacuated. No bleeding source was found. The patient made a good recovery and was discharged on the $3 \mathrm{rd}$ postoperative day.
\end{abstract}

Keywords: Extrapleural hematoma; Thoracic trauma

\section{Introduction}

Blunt thoracic trauma usually causes various injuries such as rib fractures, pneumothorax, hemothorax, and pulmonary contusion. Most of these injuries can be definitely diagnosed by a posteroanterior chest radiograph. Rarely, chest trauma may result in a collection of blood between the parietal pleura

\footnotetext{
Manuscript accepted for publication January 22, 2013

adDepartment of Surgery, St. Martin De Porres Hospital, Chiayi, Taiwan, R.O.C.

${ }^{\mathrm{b}}$ Department of Surgery, Chi Mei Medical Center, Liouying, Taiwan, R.O.C.

${ }^{\mathrm{c} C}$ Corresponding author: Yueh-Feng Tsai, Department of Surgery, St. Martin De Porres Hospital, No. 565, Sec. 2, Daya Rd., Chiayi City 600, Taiwan, R.O.C. Email: tyf5826@gmail.com
}

doi: http://dx.doi.org/10.4021/jmc1071w and the endothoracic fascia, termed extrapleural hematoma [1]. A large extrapleural hematoma appears as an opacity of the affected hemithorax on the chest X-ray mimicking a hemothorax. We report a case of huge extrapleural hematoma in relation to a fracture of the left 4 th rib after blunt trauma. The patient was initially diagnosed as a massive hemothorax. Although the differential diagnosis between an extrapleural hematoma and a hemothorax has been challenging, the careful evaluation of clinical and radiographic findings may lead to the full characterization of extrapleural hematomas.

\section{Case Report}

A 50-year-old man presented with blunt thoracic trauma after a motorcycle accident. The chest radiograph showed a fracture of the left 4th rib (Fig. 1) (arrow). Because the patient's past history included alcoholic liver cirrhosis and the platelet count was $9.2 \times 10^{4} / \mu \mathrm{L}$, we considered that he was at risk

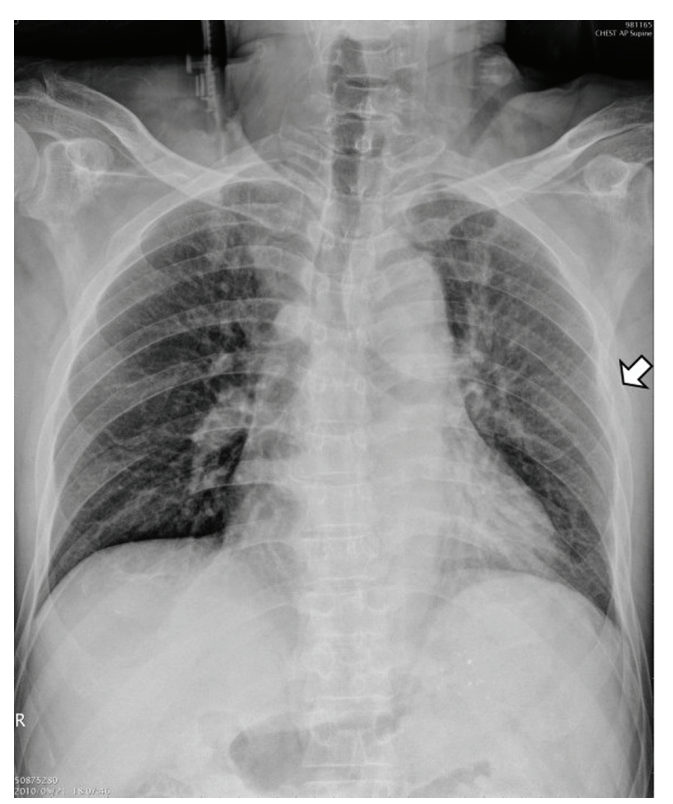

Figure 1. Chest radiograph on admission shows a fracture of the left 4th rib (arrow). 


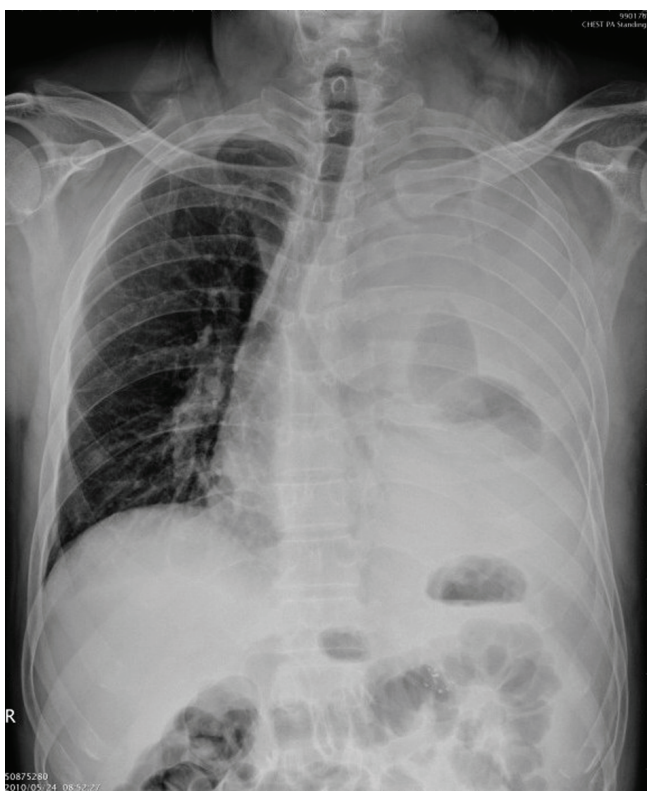

Figure 2. Complete opacity of the left hemithorax with a shift of the mediastinum to the right 3 days later.

to have delayed hemothorax. Therefore, he was admitted to the Chi Mei Liouying Hospital for observation. Three days later, worsening chest pain and shortness of breath occurred. A repeated chest X-ray showed a complete opacity of the left hemithorax with a shift of the mediastinum to the right (Fig. 2). The patient was reported to have a massive left hemotho-

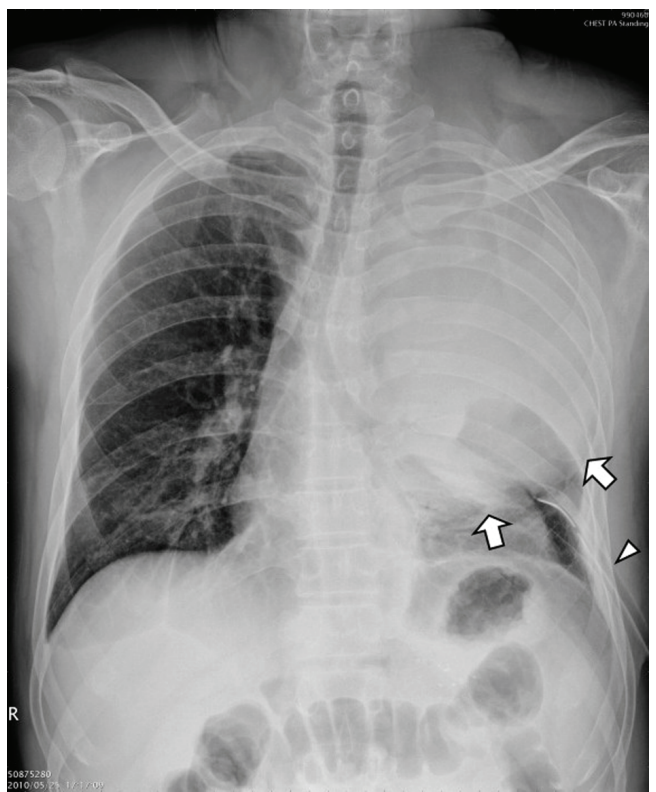

Figure 3. The left hemithorax still appears as a near complete opacity after insertion of a chest tube (arrowhead). The pleural reflection is identified at the lower margin (arrows) and the costophrenic angle is not obliterated.

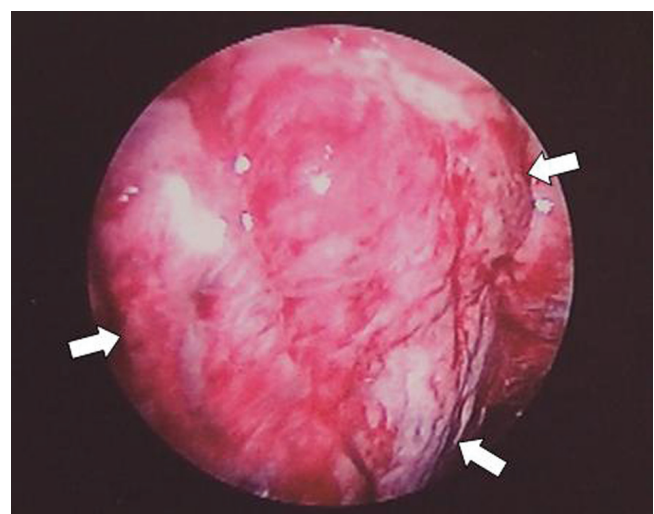

Figure 4. Video-assisted thoracic surgery shows no traumatic pleural collections but a huge pleural mass (arrows).

rax. Tube thoracostomy was performed immediately. However, only a small amount of blood-like fluid was drained and the left hemithorax still appeared as a near total opacity on the follow-up chest radiograph (Fig. 3). It is considered that the intrapleural collections were too viscous to be drained, and therefore the diagnosis of massive clotted hemothorax was made. Surprisingly, an emergency VATS revealed no traumatic pleural collections but a huge pleural mass (Fig. 4) containing a large amount of dark, reddish blood clots. The blood clots were evacuated. No bleeding source was found. The patient made a good recovery and was discharged on the 3 rd postoperative day.

\section{Discussion}

Extrapleural hematomas caused by blunt thoracic trauma have been reported mostly in isolated case reports. The incidence might be underestimated for lack of awareness of this unusual entity. Investigators reported the incidence of traumatic extrapleural hematoma was $7.1 \%$, more common than previously reported [2]. In blunt trauma patients, fractured ribs can result in lacerations of the vasculature of the chest wall, especially the intercostal vessels. If the parietal pleura is not torn or the tear is relatively small, blood is unable to flow into the pleural space and will collect in the extrapleural space causing an extrapleural hematoma [3].

The typical radiographic feature of an extrapleural hematoma is a D-shaped opacity with its base located against the corresponding part of the chest wall [2]. However, this typical D-shaped outline may not be identified in a huge extrapleural hematoma which tends to appear as a near complete opacity of the hemithorax mimicking a hemothorax. As seen in this patient, a completely opacified left hemithorax was initially diagnosed as a massive traumatic hemothorax.

It is difficult to differentiate a huge extrapleural hematoma from a hemothorax but several clinical and radiographic findings can help to make the diagnosis. In a trauma patient 
with an opacity of the hemithorax on a chest radiograph, unsuccessful drainage of collections after insertion of a chest tube may suggest an extrapleural hematoma or a clotted hemothorax. However, unlike a hemothorax, the pleural reflection may be identified at the lower margin and the costophrenic angle is not obliterated in an extrapleural hematoma (Fig. 3) $[1,3,4]$.

Although most reported cases of extrapleural hematoma could be diagnosed only using the chest radiograph, contrastenhanced computed tomography (CT) is useful to determine the location and nature of the extrapleural collections and aid preoperative diagnosis [4]. In this patient, a chest CT scan was not performed because this diagnosis was not suspected preoperatively.

Treatment of extrapleural hematomas closely depends on the clinical condition of patients. A stable patient and a small hematoma can be managed conservatively. Evacuation of blood clots may be required in a large extrapleural hematoma because respiratory and circulatory disturbances may occur [5]. Due to poor visualization and prolonged surgical time, Rashid suggested video-assisted thoracic surgery was not suited to approach the extrapleural hematoma, so that a limited thoracotomy was necessary [6]. However, in this patient VATS has been shown to be effective in exploring the pleural space and evacuating the hematoma. We believe the successful rate of VATS can be further improved by using a CT scan to determine the location of the extrapleural hematoma.
In conclusion, we report a rare case of huge extrapleural hematoma secondary to blunt trauma mimicking a hemothorax on the chest radiograph. When tube thoracostomy drainage is poor in cases of 'hemothorax', extrapleural hematoma should be included in the differential diagnosis.

\section{References}

1. Hii CH, Huong SS, Lo SE, Chiang YH, Tan CK. Extrapleural haematoma: a diagnostic pitfall in blunt chest trauma. Resuscitation. 2008;79(3):348-349.

2. Rashid MA, Wikstrom T, Ortenwall P. Nomenclature, classification, and signficance of traumatic extrapleural hematoma. J Trauma. 2000;49(2):286-290.

3. Goh BK, Koong HN. Massive traumatic extrapleural hematoma mimicking hemothorax: a potential pitfall of penetrating chest trauma. J Trauma. 2006;61(4):995997.

4. Jeong JY, Lee J. Huge extrapleural hematoma by blunt trauma mimicking traumatic hemothorax. J Trauma Acute Care Surg. 2012;72(4):E118.

5. Poyraz AS, Kilic D, Gultekin B, Ozulku M, Hatipoglu A. Extrapleural hematoma: when is surgery indicated? Monaldi Arch Chest Dis. 2005;63(3):166-169.

6. Rashid MA. Value of video-assisted thoracic surgery in traumatic extrapleural hematoma. Thorac Cardiovasc Surg. 1999;47(4):255-257. 